\title{
BG-12 in Multiple Sclerosis
}

\author{
J. Theodore Phillips, MD, $\mathrm{PhD}^{1}$ Robert J. Fox, $\mathrm{MD}^{2}$ \\ ${ }^{1}$ Multiple Sclerosis Program, Baylor Institute for Immunology \\ Research, Dallas, Texas \\ 2 Department of Neurology and Neurological Institute, Mellen Center \\ for Multiple Sclerosis Treatment and Research, Cleveland Clinic, \\ Cleveland, Ohio
}

Semin Neurol 2013;33:56-65.

\begin{abstract}
Address for correspondence J. Theodore Phillips, MD, PhD, Multiple Sclerosis Program, Baylor Institute for Immunology Research, Dallas, TX 75204 (e-mail: ted.phillips@baylorhealth.edu).
\end{abstract}

\begin{abstract}
\section{Keywords}

- multiple sclerosis

- BG-12

- dimethyl fumarate

- fumaric acids

- NF-E2-related factor 2

Dimethyl fumarate (DMF) is an orally administered agent that has been used for over 40 years for the treatment of psoriasis. Recent work demonstrates both DMF immunomodulatory and neuroprotective actions in vitro and in animal models of autoreactive central nervous system inflammation and neurodegeneration. DMF acts through chemical modification of the repressor protein Keap1, allowing stabilization and nuclear translocation of the transcription factor Nrf2, with subsequent downstream activation of a cascade of several cytoprotective and antioxidant pathways. Additionally, suppression of transcription factor NF-kB-mediated proinflammatory signaling results in the inhibition of proinflammatory responses and induction of anti-inflammatory cytokines. BG-12 is an orally administered, enteric-coated microtablet preparation of DMF. In two phase III, relapsing-remitting multiple sclerosis (MS) trials, BG-12 led to a 44 to $53 \%$ reduction in annualized relapse rate and a 71 to $85 \%$ reduction in new $T 2$ lesions on magnetic resonance imaging. The most common side effects of BG-12 are cutaneous flushing and gastrointestinal symptoms, with the highest incidence in the first month after starting treatment. No serious safety signals were seen during the phase II and III trials, including no increased risk of opportunistic infections or cancer. Altogether, BG12 's novel mechanism of action appears to provide a favorable balance of efficacy, safety, and tolerability for treatment of relapsing MS.
\end{abstract}

For almost 20 years, disease-modifying therapies have been available for treatment of relapsing forms of multiple sclerosis (MS). The current standard, first-line therapies for MS are interferon beta -1 (IFN $\beta-1$; Avonex, Biogen Idec, Weston, Massachusetts; Betaseron/Betaferon, Bayer HealthCare, Leverkeusen, Germany; Extavia, Novartis, Basel, Switzerland; Rebif, EMD Serono, Inc., Rockland, Massachusetts) and glatiramer acetate (GA; Copaxone, Teva Pharmaceuticals, Petah Tikva, Israel). Phase III clinical trials found each to reduce the rate of clinical relapses by approximately $30 \%$ relative to placebo. ${ }^{1-4}$ Side effects include skin reactions (predominantly subcutaneous therapies) and flu-like side effects and hepatic irritation (IFN $\beta-1$ therapies). A distinct advantage of these therapies is their excellent long-term safety and reasonable tolerability, although their efficacy is modest and all are administered by injection (subcutaneous or intramuscular).

Recently, three additional therapies have emerged as important long-term disease-modifying therapies for relapsing MS. Natalizumab (Tysabri, Biogen Idec, Weston, Massachusetts) is a monoclonal antibody that inhibits leukocyte trafficking into the central nervous system (CNS). Natalizumab has excellent efficacy in reducing clinical relapses, new brain lesions on magnetic resonance imaging (MRI), and delaying sustained progression of disability. ${ }^{5,6}$ Two drawbacks of natalizumab include its monthly intravenous administration and its association with progressive multifocal leukoencephalopathy (PML), a potentially fatal CNS infection. ${ }^{7}$ Fingolimod (Gilenya, Novartis, Basel, Switzerland), a second contemporary therapy, is a sphingosine receptor
Issue Theme Current and Emerging Therapies for Multiple Sclerosis; Guest Editor, B. Mark Keegan, MD, FRCP(C)
Copyright (c) 2013 by Thieme Medical Publishers, Inc., 333 Seventh Avenue, New York, NY 10001, USA. Tel: +1(212) 584-4662. 
modulator that both reduces leukocyte egress from peripheral lymphoid tissue and may have direct immunomodulating effects within the CNS. ${ }^{8}$ Fingolimod shows beneficial relapse, disability progression and MRI effects. A head-tohead study showed fingolimod is more effective than IFN $\beta$ $-1 .^{9}$ However, fingolimod is associated with cardiac side effects (mostly initial bradycardia events), macular edema, and increased risk of some infections (most notably respiratory infections and herpes virus infections). A third therapy, teriflunomide (Aubagio, Genzyme, Cambridge, Massachusetts) has been recently approved for relapsing MS in the United States. Teriflunomide inhibits new pyrimidine synthesis and rapid proliferation of activated lymphocytes through its action on dihydroorotate dehydrogenase. ${ }^{10}$ Teriflunomide shows beneficial relapse, disability progression, and MRI effects. Gastrointestinal disturbances, hair thinning, and mild-moderate elevations in alanine aminotransferase levels have been noted more commonly with teriflunomide than placebo. Based on animal studies, the U.S. Food and Drug Administration (FDA) has also issued an alert concerning possible teratogenic effects in humans. ${ }^{11}$

Despite the availability of many disease-modifying therapies for relapsing MS, none have the ideal treatment triad: strong efficacy, good tolerability, and excellent safety. The BG12 preparation of dimethyl fumarate (DMF) may come closer to offering that ideal treatment triad.

\section{Initial Clinical Experience with Fumaric Acids}

Fumaric acid (FA) is an intermediate in the citric acid cycle used by cells to produce energy. In 1946, food chemists recognized its acid regulatory properties and fruit-like taste. FA is still routinely used as a food acidulent in beverages, baking powders, and candy (-Table $\mathbf{1}$ ).
The medicinal use of fumaric acids (FA) is credited to German chemist Walter Schweckendiek, who suffered from psoriasis. In the late 1950s, Schweckendiek turned to his professional expertise in chemistry to search for treatments for his skin condition. He proposed that psoriasis resulted from a defect in carbohydrate metabolism involving the citric acid cycle and its oxidative steps, and predicted improvement through ingestion of FA. ${ }^{12}$ However, his first treatment attempts with oral FA were disappointing owing to significant gastrointestinal irritation. To improve gastrointestinal absorption and tolerability, Schweckendiek ${ }^{13}$ synthesized a mixture of FA ester (FAE) salts that did prove effective in treating his psoriasis, and also demonstrated less irritant side effects. Gunther Schafer, a general practitioner, later promoted a standardized treatment regimen using an FAE mixture and restricted diet, and subsequently reported encouraging open-label results with over $70 \%$ of 900 psoriasis patients showing improvement. ${ }^{14}$ Despite the lack of data supporting the metabolic defect hypothesis, efficacy and safety of FAE in psoriasis were subsequently demonstrated in two randomized, double-blinded, placebo-controlled studies. ${ }^{15,16}$ These studies were performed using a proprietary preparation of FAE (120 mg dimethyl fumarate [DMF], $87 \mathrm{mg}$ monoethyl fumarate calcium salt, $5 \mathrm{mg}$ monoethyl fumarate magnesium salt, and $3 \mathrm{mg}$ monoethyl fumarate zinc salt per entericcoated tablet). In 1994, the Swiss company Fumapharm (acquired by Biogen Idec, Weston, Massachusetts) received German regulatory approval for this FAE preparation (called Fumaderm) for the treatment of psoriasis. Fumaderm is currently one of the most widely prescribed systemic therapies for psoriasis in Germany, with visible improvement typically noted within 6 weeks. Fumaderm is generally well tolerated; cutaneous flushing and gastrointestinal upset are the most commonly reported side effects. A small study has

Table 1 Timeline of development of BG-12/DMF in multiple sclerosis

1946: Fumaric acids are recognized by food chemists for their acid regulatory properties and fruit-like taste.

1960s-1980s: German chemist Walter Schweckendiek and general practitioner Gunther Schafer develop fumaric acid esterbased regimen to treat psoriasis. ${ }^{12,14}$

1994: The Swiss company Fumapharm receives German regulatory approval for Fumaderm, which is a proprietary formulation of dimethyl fumarate and several fumarate ester salts.

2000: Bochum neurologist Horst Przuntek observes that MS patients with psoriasis appear to have stabilized MS after starting Fumaderm.

2002: A patent is filed for the use of fumaric acids in the treatment of MS.

2003: Fumapharm establishes collaboration with Biogen Idec to develop fumaric acids in MS.

2006: Schilling and colleagues show DMF or MMF effectively prevents chronic EAE. ${ }^{24}$

2006: Schimrigk and colleagues publish a case series of $10 \mathrm{MS}$ patients treated with Fumaderm. ${ }^{51}$

2007: A placebo-controlled phase II trial shows a robust reduction in MRI lesions with BG-12,70 and two phase III trials in relapsing remitting MS get underway. ${ }^{52,53}$

2011: Linker and colleagues demonstrate fumaric acid esters activate the Nrf-2 antioxidant pathway and exert neuroprotective effects in EAE-associated neuroinflammation. ${ }^{25}$

2011-2012: Both phase III trials report positive results on clinical and MRI outcomes. ${ }^{52,53}$

Abbreviations: DMF, dimethyl fumarate; EAE, experimental autoimmune encephalomyelitis; MMF, monomethyl fumarate; MRI, magnetic resonance imaging; MS, multiple sclerosis. 
found acceptable long-term safety in psoriasis patients treated for 10 to 14 years. $^{17}$

\section{DMF Mechanisms of Action}

\section{Pharmacokinetics}

Comparative studies have shown that DMF (C6H8O4; MW 141.1) is the key FAE component accounting for therapeutic benefit in psoriasis. ${ }^{18}$ DMF is rapidly hydrolyzed to monomethyl fumarate (MMF; also known as MHF, methylhydrogen fumarate) by esterases in the alkaline environment of the small intestine. ${ }^{19}$ In healthy volunteers and psoriasis patients, time to detectable MMF in blood is somewhat delayed with food intake, but without effect on maximum blood concentration 5 to 6 hours later. ${ }^{20}$ Plasma FA levels remain unchanged by FAE ingestion. MMF is detectable in blood after a variable period of 60 minutes or more, whereas DMF is subject to a strong first-pass metabolism and is not systemically detectable at any time after oral DMF ingestion. ${ }^{20,21}$ MMF is further metabolized ( $50 \%$ protein-bound; serum halflife $36 \mathrm{~h}^{22}$ ) in the citric acid cycle to yield water and carbon dioxide. DMF is highly lipophilic and may be absorbed by intestinal tissue, where it is at least partially metabolized to MMF. DMF can also conjugate directly to intracellular glutathione and be released systemically as a (possibly bioactive) glutathione conjugate, which is further metabolized to DMFmercapturic acid and detectable in urine. ${ }^{23}$ Additionally, DMF may also be released unaltered into the portal circulation and taken into venous blood cells, or hydrolyzed to MMF in the plasma. These scenarios suggest that DMF could still have biologically relevant activities in vivo (in addition to MMF) despite undetectable free-plasma DMF after ingestion. A small amount of unaltered MMF is excreted in urine and feces. FAEs do not appear to be nephrotoxic. Cytochrome p450-dependent pathways are not involved in FAE metabolism, and drug interactions have not been reported.

\section{In Vivo Effects of DMF and Experimental Models of Demyelination and Neurodegeneration}

Efficacy of FAEs in the prevention of myelin-oligodendrocyte glycoprotein (MOG)-induced experimental autoimmune encephalomyelitis (EAE) in mice was first identified in $2006 .{ }^{24}$ MOG-EAE is an animal model of inflammatory demyelination that also displays features resembling several neurodegenerative aspects of MS. Either DMF or MMF given preventatively in acute MOG-EAE led to decreased CNS inflammatory infiltrates and increased serum interleukin10 (IL-10; an anti-inflammatory cytokine). Linker and colleagues later confirmed the preventative effects of DMF given during the acute phase of MOG-EAE, and extended these findings to demonstrate a therapeutic effect in established, chronic MOG-EAE. ${ }^{25}$ However, in contrast to findings in acute phase EAE, DMF did not significantly decrease inflammatory infiltrates when administered during the chronic phase of MOG-EAE. Interestingly, clinical benefit in this model appeared to result primarily from reduction in demyelination with relative preservation of myelin and axons. This interesting, but unexpected, outcome was suggested to reflect the rescue of neurons and glial (astrocytes) cells from oxidative stress-induced cell death through neuroprotection that is mediated by DMF-induced activation of the nuclear factor(erythroid-derived 2-) related factor-2 (Nrf2) pathway. Similarly, other studies have now also demonstrated DMFinduced, Nrf2-dependent cytoprotection of neurons and astrocytes. $^{26-28}$

Evidence of oxidative stress is readily apparent within the CNS in EAE and MS. ${ }^{29-31}$ Glutathione, a major intracellular reactive oxygen species (ROS) scavenger, is decreased within CNS inflammatory foci, ${ }^{32}$ and a variety of antioxidant proteins, including Nrf2, are increased in MS lesions. ${ }^{33}$ The importance of the Nrf2 pathway in regulating CNS inflammation is emphasized by the observation of significantly worsened EAE in Nrf2 $(-/-)$ knockout mice. ${ }^{25,34}$ Also, DMF neuroprotective effects are not inducible in $\mathrm{Nrf2}(-/-)$ mice.

The effects of DMF or MMF have also been examined in other models of demyelination and neurodegeneration. In the cuprizone model of toxic, noninflammatory demyelination, DMF or MMF demonstrated minimal or no significant impact on demyelination or remyelination. ${ }^{35}$ In contrast to EAE, no obvious protective effects of DMF or MMF on CNS cellular elements were found in this model. However, in an experimental model of neurodegeneration with similarities to Huntington's disease, the transgenic mouse strains R6/2 and YAC128 showed preservation of striatal and motor cortex neurons and improved short- and long-term motor outcomes with DMF treatment. ${ }^{36}$ Apparent DMF-associated neuroprotection has also been observed in a rat model of excitotoxic neurodegeneration induced by intrastriatal malonate injection. ${ }^{37}$ In this model, DMF treatment resulted in reduction of striatal lesion volume and improved abnormal apomorphineinduced rotational behavior compared with vehicle controls.

\section{DMF Effects on the Immune System}

After dismissal of the initial citric acid cycle defect hypothesis, early explanations of FAE therapeutic effects focused on the proinflammatory T-cell (Th1) theory of certain autoimmune disorders, including psoriasis ${ }^{38}$ and $\mathrm{MS}^{39}$ In addition to antiproliferative effects on keratinocytes, ${ }^{40}$ FAEs were found to have direct effects on multiple components of the immune system. MMF induces anti-inflammatory cytokines IL-4, IL-5, IL-10, and IL-1RA production by human peripheral blood mononuclear cells, without stimulation of proinflammatory cytokines IL-2, interferon gamma (IFNY), or IL-12. ${ }^{41-43}$ Other studies found that DMF or MMF may decrease production of IFNr and IL-12, ${ }^{44}$ as well as decrease expression of cytokineinduced endothelial cell adhesion molecules ICAM-1, VCAM1 , and E-selectin. ${ }^{45}$ In vitro differentiation of antigen-presenting, monocyte-derived dendritic cells ( $\mathrm{mDCs}$ ) is inhibited by DMF or MMF, resulting in decreased $\mathrm{MDC}$ production of IL6, IL-10, IL-12, TNF $\alpha$, and decreased T-cell production of proinflammatory IFN $\gamma$ and IL-17. ${ }^{46-49}$

DMF, but not MMF, may induce apoptosis in cultured mDCs and activated T cells. ${ }^{50}$ Possibly related, a transient $4 \%$ increase in CD4+ T cell apoptosis was reported in Fumaderm-treated RRMS patients during the first 12 weeks of therapy. ${ }^{51}$ Apoptosis may therefore explain in part the mild decrease in circulating 
lymphocyte count observed in psoriasis ${ }^{16}$ and $\mathrm{MS}^{52,53}$ patients treated with Fumaderm or BG-12, respectively.

\section{Subcellular Mechanisms of Action}

Twenty years ago, a systematic study of different compounds capable of inducing phase II detoxifying enzymes (e.g., NAD $(\mathrm{P}) \mathrm{H}$ quinone oxidoreductase-1; NQO1) identified DMF, among others, to be highly effective. ${ }^{54}$ A common structural feature among these inducing compounds is that of a socalled Michael acceptor, which reflects the compound's relatively electrophilic nature. As such, DMF and several other Michael acceptor compounds were shown to activate an electrophile-responsive element (EpRE) located in the 5' flanking region of the mouse glutathione S-transferase Ya subunit gene. ${ }^{55}$ EpRE is also known, perhaps more commonly, as ARE (antioxidant-responsive element). ${ }^{56,57}$

Further work showed that the effect of DMF and MMF on ARE/EpRE is actually indirect. Under normal conditions, ARE/ EpRE is not active; however, under cellular stress conditions, Nrf2 activates ARE/EpRE. ${ }^{58} \mathrm{Nrf} 2$ is normally not available for downstream regulatory interactions as it is targeted for rapid ubiquitin E3 ligase-(Cul3-) directed proteasomal degradation through its usual bound interaction with the cytosolic repressor Keap1 (Kelch-like ECH-associated protein 1). DMF or MMF S-alkylates Cys151 of cysteine-rich Keap1, thereby blocking the interaction of Keap1 and Nrf2, which prevents Nrf2 ubiquitination and leads to Nrf2 stabilization, phosphorylation, nuclear translocation, and subsequent Nrf2 nuclear activation of ARE/EpRE. ${ }^{59}$ Therefore, Keap1 can be considered a critical intracellular redox sensor; therefore, it is sensitive to inorganic and organic hydroperoxides, peroxynitrite, and other electrophilic molecular species generated by oxidative stress and tissue damage. ${ }^{60}$ ARE/EpRE activation results in an increase in synthesis of many antioxidation-related proteins such as NQO1, heme oxygenase-1 (HO-1), glutamate-cysteine ligase catalytic subunit (GCLC), glutamate-cysteine ligase modifier subunit (GCLM), peroxiredoxin-1 (Prx1), glutathione S-transferase Mu-1 (GSTM1), thioredoxin (Trx), thioredoxin reductase (TrxR), and various heat shock proteins. ${ }^{60}$ ARE/EpRE activation also enhances other prosurvival processes such as phase II detoxification, nucleotide excision repair, and autophagy inhibition. $\mathrm{Nrf} 2$ signaling is ultimately terminated through a combination of Nrf2 nuclear export processes dependent on Fyn-mediated Nrf2 phosphorylation, as well as removal of Nrf2-activating signals through the action of the induced antioxidant enzymes. ${ }^{60}$

DMF and MMF also enhance glutathione synthesis through the Nrf2 signaling pathway, despite initial reduction of intracellular glutathione stores through direct reaction with glutathione. ${ }^{26-28,61-64}$ Importantly, initial glutathione reduction in mDCs results in preferential induction of type II mDCs promoting Th2 differentiation, and also blocks STAT1 phosphorylation, thereby reducing proinflammatory mDC IL-12 production. ${ }^{49}$

NF-kB, a key redox-sensitive, proinflammatory nuclear transcription factor, is inhibited by DMF or MMF through Nrf2-mediated induction of phase II antioxidants, with subsequent reduction of intracellular ROS accumulation in im- mune-activated cells, and inhibition of NF-kB upstream signal mediators Akt, NIK, and IKK. ${ }^{60,65}$ Through these indirect inhibitory actions of DMF and MMF on NF-kB, proinflammatory gene expression is suppressed. DMF also inhibits NF-kB activity through suppression of ERK1/2 and downstream MSK1 kinase activities, and subsequent reduction of p65 (RelA) phosphorylation, nuclear translocation, and target gene transactivation. ${ }^{48,66}$ In addition, recent work suggests that DMF may be capable of suppressing inflammation through one or more Nrf2-independent pathways. ${ }^{67}$

MMF, but not DMF, also competitively binds and activates the high-affinity nicotinic acid (NA) receptor GPR109A, with subsequent production of prostanoids. ${ }^{68}$ This interaction appears to account for commonly encountered MMF- (and NA-) induced cutaneous flushing. COX-1 and COX-2 inhibitors were found to decrease early- and late-phase flushing, respectively, which prompted a recent successful study with aspirin to lessen flushing after DMF ingestion. ${ }^{69}$

\section{Summary of DMF Mechanisms of Action}

DMF is a small electrophilic molecule that is rapidly converted in the gut to MMF, its major bioactive metabolite. MMF alkylates the intracellular redox sensor Keap1, resulting in stabilization and nuclear translocation of Nrf2, which in turn activates the gene regulatory element ARE/EpRE with subsequent downstream induction of a variety of antioxidant and cytoprotective proteins. Through reduction of intracellular ROS by several of these induced phase II proteins, proinflammatory activities of NF-kB are reduced. DMF direct effects on lymphocytes and dendritic cells induce Nrf2-related pathways, inhibit proinflammatory cytokine production, and promote a shift from Th1 (and Th17) to Th2-like immune activities. Other non-Nrf2 mediated pathways may be involved in DMF anti-inflammatory effects as well. DMF direct effects on neurons and glia (primarily astrocytes) induce Nrf2-related pathways that promote neuroprotection and cell survival. Therefore, DMF-associated net beneficial effects in MS are expected to result from combined anti-inflammatory and neuroprotective actions.

\section{Early Studies of BG-12 in Multiple Sclerosis}

The idea of using FAEs therapeutically in MS was reportedly born in the late 1990s at St. Josef Hospital, Ruhr University, Bochum, Germany, when neurology chair Horst Przuntek noticed that the disease course of two of his MS patients appeared to stabilize after they started treatment of their concomitant psoriasis with Fumaderm [R. Gold, personal communication, 2012]. His colleague Sebastian Schimrigk then conducted an open-label, phase I trial of Fumaderm in 19 MS patients, which found a 95\% reduction in number of gadolinium-enhancing $(\mathrm{Gd}+)$ lesions over 48 weeks. $^{51}$

Gastrointestinal side effects from Fumaderm led to its further refinement into BG-12, which is an enteric-coated, microtablet preparation of DMF. A U.S. patent was filed in 2002 for the use of FAE in the treatment of MS, and a formal collaboration with between Fumapharm and Biogen Idec was established in October 2003. 
Subsequently, a 48-week, 257 patient phase IIb clinical trial in relapsing-remitting MS was conducted (ClinicalTrials. gov identifier: NCT00168701). Participants were randomized to three different doses of BG-12 (120 mg daily, or $120 \mathrm{mg}$ three times daily [360 mg/d], or $240 \mathrm{mg}$ three times daily [720 mg/d]) or placebo for 24 weeks, followed by a 24 -week dose-blinded safety extension (placebo switched to BG-12 $240 \mathrm{mg}$ three times daily). ${ }^{70}$ The primary outcome was its effect on new Gd+ lesions, where the highest dose of BG-12 showed a $69 \%$ reduction $(p<0.0001)$ in lesion number compared with placebo. The middle dose group $(120 \mathrm{mg}$ three times daily) did not show a significant reduction in lesions, but was probably handicapped by a $56 \%$ higher baseline MRI disease activity relative to the placebo group. When the primary outcome is instead expressed as the within-group reduction in MRI disease activity compared with each group's prestudy baseline, a consistent dose response is observed: $29 \%, 41 \%, 69 \%$, and $73 \%$ lesion reduction in the placebo, $120 \mathrm{mg} / \mathrm{d}, 360 \mathrm{mg} / \mathrm{d}$, and $720 \mathrm{mg} / \mathrm{d}$ groups, respectively. BG-12 was tolerated relatively well, with $91 \%$ of patients completing the fully blinded first 24 weeks, and $97 \%$ completing the dose-blinded, 24-week safety extension. As seen with Fumaderm, the most common BG-12 associated adverse effects were skin flushing and gastrointestinal events.

\section{Phase III Trials of BG-12 in Multiple Sclerosis}

Based upon these encouraging phase II trial results, two phase III trials were conducted: Determination of the Efficacy and Safety of Oral Fumarate in Relapsing-Remitting Multiple Sclerosis (DEFINE; ClinicalTrials.gov identifier: NCT00420212) (33 $^{53}$ and Comparator and an Oral Fumarate in Relapsing-Remitting Multiple Sclerosis (CONFIRM; ClinicalTrials.gov identifier: NCT00451451).52 Both DEFINE and CONFIRM were 2-year, randomized, placebo-controlled trials, evaluating clinical relapses, progressive disability, and (in a subset of patients) MRI disease activity. Two doses of BG-12 were evaluated-240 mg three times per day $(720 \mathrm{mg} / \mathrm{d})$, and $240 \mathrm{mg}$ twice a day (480 $\mathrm{mg} / \mathrm{d}$ with placebo given for the midday dose)-and were compared with placebo given three times per day. The CONFIRM trial also included glatiramer acetate (GA) as an openlabel (but still randomized and evaluator-blinded) treatment arm, as required by the European Medicines Agency, to provide a risk-benefit comparison to a standard therapy. Because of potential partial treatment unblinding due to skin flushing and gastrointestinal side effects, several steps were taken to ensure study validity: blinded examining neurologists were unaware of either treatment assignment (oral therapy vs. GA) or patient; patients were instructed to not take study medication within 4 hours of each study visit to reduce potential unblinding of study investigators; an independent, blinded adjudication committee confirmed all relapses; and image analysis was conducted blinded to all study information. Enrollments of DEFINE and CONFIRM studies started in 2007, and the last patient completed follow-up in 2011. The DEFINE study enrolled and dosed 1,234 patients; the CONFIRM study enrolled and dosed 1,417 (including the additional GA arm). Both studies enrolled patients from North and Central America, Europe, and Asia. Of note, these trials were one of the first to enroll MS patients in India. Seventy-seven percent of the study participants in the DEFINE study completed the study (68\% on originally assigned study medication), and $80 \%$ of the study patients in the CONFIRM study completed the study (71\% on originally assigned study medication).

\section{Efficacy}

In the DEFINE and CONFIRM studies, both dosing regimens of BG-12 reduced the annualized relapse rate (ARR; the primary outcome in the CONFIRM study) by 44 to $53 \%$ ( $p<0.001$ for all; - Table 2)..$^{52,53}$ Various sensitivity analyses in both studies showed consistent results. Similarly, the risk of relapse (the primary outcome in the DEFINE study) was also reduced by 34 to $50 \%$ in both dosing regimens $(p<0.001$; - Table 2). BG-12 reduced the risk of sustained progression of disability by 34 to $38 \%$ in the DEFINE study $(p<0.05$ ), and 21 to $24 \%$ in the CONFIRM study, which was not significant. In the CONFIRM

Table 2 Key outcomes in BG-12 phase III studies

\begin{tabular}{|l|l|l|l|l|l|}
\hline Reduction in: & \multicolumn{2}{|c|}{ Define $^{53}$} & \multicolumn{2}{c|}{ Confirm ${ }^{52}$} & GA \\
\hline & bid (\%) & tid (\%) & bid (\%) & tid (\%) \\
\hline Annualized relapse rate & $53^{\mathrm{a}}$ & $48^{\mathrm{a}}$ & $44^{\mathrm{a}}$ & $51^{\mathrm{a}}$ & $29^{\mathrm{b}}$ \\
\hline Risk of relapse & $49^{\mathrm{a}}$ & $50^{\mathrm{a}}$ & $34^{\mathrm{b}}$ & $45^{\mathrm{a}}$ & $29^{\mathrm{b}}$ \\
\hline Risk of sustained progression of disability & $38^{\mathrm{b}}$ & $34^{\mathrm{c}}$ & $21^{\mathrm{d}}$ & $24^{\mathrm{d}}$ & $7^{\mathrm{d}}$ \\
\hline New or enlarging T2 lesions & $85^{\mathrm{a}}$ & $74^{\mathrm{a}}$ & $71^{\mathrm{a}}$ & $73^{\mathrm{a}}$ & $54^{\mathrm{a}}$ \\
\hline New T1-hypointense lesions & $72^{\mathrm{a}}$ & $63^{\mathrm{a}}$ & $57^{\mathrm{a}}$ & $65^{\mathrm{a}}$ & $41^{\mathrm{b}}$ \\
\hline New gadolinium-enhancing lesions & $90^{\mathrm{a}}$ & $73^{\mathrm{a}}$ & $74^{\mathrm{a}}$ & $65^{\mathrm{a}}$ & $61^{\mathrm{a}}$ \\
\hline
\end{tabular}

Abbreviations: BID, twice daily dosing; GA, glatiramer acetate; TID, three times daily dosing.

Note: Main clinical and MRI outcomes from the DEFINE (Determination of the Efficacy and Safety of Oral Fumarate in Relapsing-Remitting Multiple Sclerosis) and CONFIRM (Comparator and an Oral Fumarate in Relapsing-Remitting Multiple Sclerosis) phase III clinical trials.

${ }^{\mathrm{a}} \mathrm{p}<0.001$.

${ }^{\mathrm{b}} \mathrm{p} \leq 0.01$.

${ }^{c} p<0.05$.

${ }^{\mathrm{d}}$ Not significant. 
study, only 13 to $17 \%$ of patients in each group had sustained progression of disability, which was lower than the 16 to $27 \%$ of patients with progressive disability in the DEFINE study. This difference may contribute to the difference in progression outcomes between the two studies. BG-12 also showed a robust effect on MRI measures of inflammatory disease activity in both studies: the number of new or newly enlarging T2 lesions on MRI was reduced by 71 to $85 \%$; the number of $\mathrm{Gd}+$ lesions were reduced by 65 to $90 \%$, and new T1 black holes were reduced by 57 to $72 \%$ (all $p<0.001$; - Table 2 ).

Compared with placebo, GA performed similarly to that seen in previous clinical trials, ${ }^{4,71}$ with a $29 \%$ reduction in ARR and a $29 \%$ reduction in the risk of relapse. The proportion with sustained progression of disability was not different from placebo ( $7 \%$ reduction, $p=0.70$ ). GA reduced new or newly enlarging T2 lesions by $54 \%(p<0.001), \mathrm{Gd}+$ lesions by $61 \%(p<0.001)$, and T1 black holes by $41 \%(p=0.002)$. Compared with placebo, the treatment effect of BG-12 was numerically greater than GA for all clinical and imaging outcomes. In a post hoc direct comparison, BG-12 showed a greater treatment effect than GA on ARR (three times daily only, $p=0.02$ ); new or enlarging T2 lesions (twice daily, $p=0.007$; three times daily, $p=0.002$ ); and new T1 lesions (three times daily only, $p=0.003$ ).

Importantly, except for the additional GA treatment arm in the CONFIRM study, both the DEFINE and CONFIRM studies were identically designed, with the same inclusion and exclusion criteria, treatment assignments, clinical management, safety monitoring, and outcome criteria. This identical study design allowed a subsequent, preplanned combined analysis of both studies, comparing each BG-12 dose to placebo. This combined analysis found a reduction in the proportion of patients with sustained progression of disability of $32 \%$ (BG-12 twice daily, $p=0.003$ ) and 30\% (BG-12 three times daily, $p=0.006) .{ }^{72}$ In addition, the combined analysis showed a $49 \%$ reduction in ARR with both BG-12 dosing regimens ( $p<0.0001$ ), and a reduction in the risk of relapse of $43 \%$ (twice daily, $p<0.0001$ ) and $47 \%$ (three times daily, $p<0.0001$ ). MRI lesion reduction in the combined analysis was similarly robust, with 73 to $78 \%$ reduction in new or enlarging T2 lesions ( $p<0.0001$ for both doses), 64 to $65 \%$ reduction in new T1 lesions ( $p<0.0001$ ), and 70 to $83 \%$ reduction in the odds of having a greater number of $\mathrm{Gd}+$ lesions vs placebo $(p<0.0001) .^{73}$

\section{Safety and Tolerability}

BG-12 was generally well tolerated in both phase III trials, with skin flushing and gastrointestinal events reported as the most common BG-12 associated adverse events. Skin flushing was reported in 28 to $38 \%$ of BG-12 treated patients in both studies, compared with 5 to $6 \%$ of placebo patients. Skin flushing occurs approximately 30 to 45 minutes after dosing, typically involves the chest, neck, and face, and usually resolves spontaneously after 15 to 30 minutes. In a small study with healthy volunteers, aspirin was shown to almost completely block BG-12-related flushing. ${ }^{69}$ Gastrointestinal events were reported in 36 to $41 \%$ of BG-12 treated patients in both studies, compared with $26 \%$ of placebo patients. These gastrointestinal events include nausea, vomiting, abdominal pain, and diarrhea. Flushing led to treatment discontinuation in 1 to $4 \%$ of BG-12 treated patients, and nausea or diarrhea each led to treatment discontinuation in 0 to $2 \%$ of BG-12 treated patients. The incidence of both flushing and gastrointestinal events peak in the first month of treatment, and significantly decline thereafter. The incidence of serious adverse events was similar between BG-12 groups and placebo. There was an equal, low rate of cancer across placebo and treatment groups in both studies.

Infections were observed in 56 to $68 \%$ of BG-12 treated patients, compared with 50 to $65 \%$ of placebo-treated patients in both studies. The incidence of serious infections was 1 to $2 \%$ across all groups. No opportunistic infections were reported in BG-12-treated patients. There was a reduction in mean white cell count (11-12\%) and mean lymphocyte count (28$32 \%$ ) in both BG-12 treatment groups during the first year, which then plateaued and remained within the normal range. White blood cell counts $<3.0 \times 10^{9} / \mathrm{L}$ were seen in 7 to $10 \%$ of BG-12-treated patients (and 1\% of placebo), while lymphocyte counts $<0.5 \times 10^{9} / \mathrm{L}$ were seen in 4 to $5 \%$ of BG-12treated patients (and $<1 \%$ of placebo); none of these patients were discontinued from BG-12 due to these laboratory values. An increased incidence of mildly elevated liver aminotransferase levels was seen during the first few months of treatment in the DEFINE (6\% in BG-12 groups, and 3\% in placebo) study, but not in the CONFIRM study.

\section{Ongoing Studies}

At the conclusion of both the DEFINE and CONFIRM studies, patients who successfully completed 2 years of follow-up were offered enrollment in an open-label extension study. Patients originally randomized to BG-12 continued their previous regimen (twice daily or three times daily), and patients originally randomized to placebo or GA were rerandomized to either BG-12 twice daily or BG-12 three times daily. Follow-up includes clinical, MRI, and safety assessments. Although regulatory submission included only twice daily dosing, the inclusion of three times daily in the longterm follow-up will identify whether the three times daily regimen may have long-term benefits over a twice daily regimen that were not apparent over the first 2 years of treatment.

In addition, a 6-month, open-label, add-on study has been conducted in patients receiving either IFN $\beta-1$ or GA, intended primarily to evaluate the safety of adding BG-12 to standard first-line disease modifying therapies. This combination study is complete, although results have not yet been reported.

\section{Discussion}

BG-12 shows robust efficacy in relapsing MS as measured by relapse rate, disability progression, and new lesions on MRI. Although a significant slowing in sustained progression of disability was shown only in the DEFINE study, a preplanned combined analysis of DEFINE and CONFIRM showed that both 


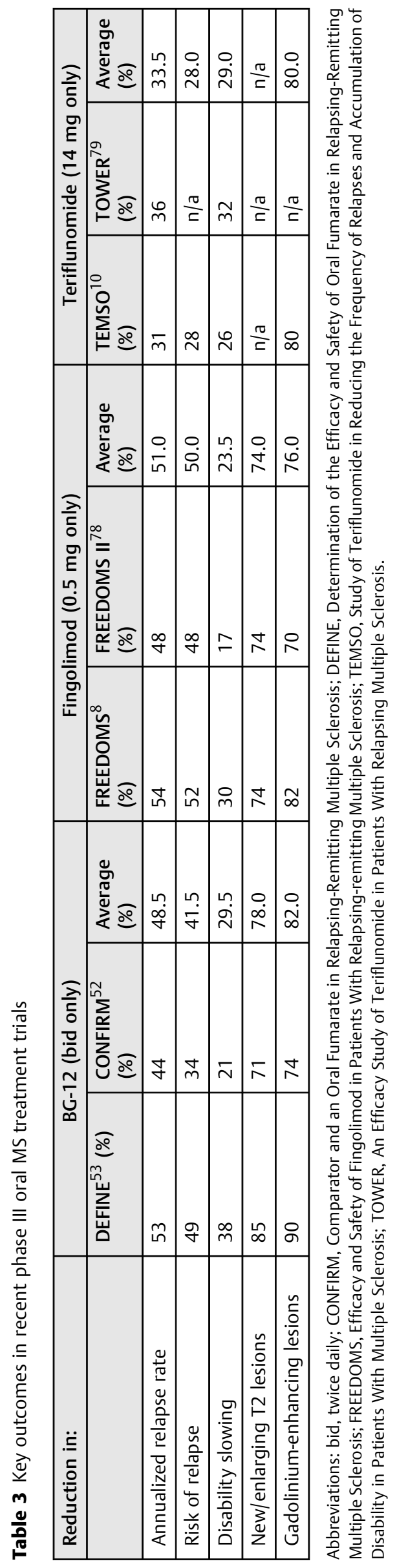

BG-12 regimens significantly slowed the progression of disability relative to placebo. BG-12 was also relatively well tolerated, with skin flushing and gastrointestinal events being the most common adverse events, leading to only a small proportion of treatment discontinuations. The incidence of these adverse events was maximal in the first month and declined thereafter, emphasizing the importance of patient education and early clinical follow-up after starting BG-12. The safety profile of BG-12 appears strong. BG-12 has been associated with only a slight increased incidence of infections (i.e., upper respiratory tract infections) and no increased incidence of opportunistic infections. Mild hepatic enzyme elevations were seen with BG-12 in the DEFINE study, but not the CONFIRM study. There was no increase in cancer in either study.

BG-12 also showed favorable efficacy compared with GA. Although the CONFIRM trial was neither designed nor powered to demonstrate superiority or noninferiority of BG-12 compared with GA, the magnitude of efficacy favored both doses of BG-12 across the majority of endpoints. Furthermore, the efficacy and safety of GA was generally consistent with results from its previous studies in relapsing MS, ${ }^{4,71}$ thus supporting the validity of the CONFIRM study results.

It is of interest to compare the efficacy of BG-12 with that of two other recently approved oral MS therapies: fingolimod and teriflunomide. All three therapies are oral and are generally well tolerated, which provide distinct advantages compared with the current injectable therapies. Each of these oral therapies has been evaluated in multiple placebocontrolled phase III trials in relapsing MS. Although each study was conducted somewhat differently and included patients from different regions of the world, previous meta-analyses suggest that the results of placebo-controlled trials in relapsing MS can be successfully compared one to another for relative efficacy. ${ }^{74}$ Indeed, the similar results from separate phase III trials of injectable therapies (IFN $\beta-1$ and $\mathrm{GA})$ predicted the similar efficacies of these therapies when they were compared head-to-head. ${ }^{75-77}$ - Table 3 shows the results of the placebo-controlled, phase III trials of BG-12, fingolimod, and teriflunomide at a dosing currently approved or submitted for regulatory approval (240 mg twice daily for BG-12, $0.5 \mathrm{mg}$ daily for fingolimod, and $14 \mathrm{mg}$ daily for teriflunomide) ${ }^{8,10}$ The efficacy of the three therapies appears broadly comparable across clinical and imaging outcomes.

\section{Conclusion}

Despite advances in the treatment options for relapsing MS, there remains a need for an effective, safety, and welltolerated MS disease modifying therapy. BG-12 appears to approach this ideal combination, with comparable efficacy to other approved oral therapies for MS. Over 20 years of extensive clinical experience with the fumaric acids in psoriasis suggests that BG-12 may have very strong long-term safety. The mechanisms of BG-12 action are unique and appear to involve both immunomodulatory as well as separate neuroprotective components. Altogether, BG-12 appears 
to be a very promising and unique addition to the therapeutic options for relapsing MS.

\section{Acknowledgments}

Dr. Phillips receives consulting fees from Biogen Idec, Genzyme, Novartis, Sanofi, and Teva, and grant support from Roche. Dr. Fox receives consulting fees from Avanir, Biogen Idec, EMD Serono, Novartis, and Questcor, and grant support from Novartis.

\section{References}

1 Jacobs LD, Cookfair DL, Rudick RA, et al; The Multiple Sclerosis Collaborative Research Group (MSCRG). Intramuscular interferon beta-1a for disease progression in relapsing multiple sclerosis. Ann Neurol 1996;39(3):285-294

2 The IFNB Multiple Sclerosis Study Group and The University of British Columbia MS/MRI Analysis Group. Interferon beta- $1 \mathrm{~b}$ in the treatment of multiple sclerosis: final outcome of the randomized controlled trial. Neurology 1995;45(7):1277-1285

3 PRISMS Study Group. Randomised double-blind placebo-controlled study of interferon beta-1a in relapsing/remitting multiple sclerosis. PRISMS (Prevention of Relapses and Disability by Interferon beta-1a Subcutaneously in Multiple Sclerosis) Study Group. Lancet 1998; 352(9139):1498-1504

4 Johnson KP, Brooks BR, Cohen JA, et al. Copolymer 1 reduces relapse rate and improves disability in relapsing-remitting multiple sclerosis: results of a phase III multicenter, double-blind placebo-controlled trial. The Copolymer 1 Multiple Sclerosis Study Group. Neurology 1995;45(7):1268-1276

5 Polman $\mathrm{CH}$, O'Connor PW, Havrdova E, et al; AFFIRM Investigators. A randomized, placebo-controlled trial of natalizumab for relapsing multiple sclerosis. N Engl J Med 2006;354(9): 899-910

6 Rudick RA, Stuart WH, Calabresi PA, et al; SENTINEL Investigators. Natalizumab plus interferon beta-1a for relapsing multiple sclerosis. N Engl J Med 2006;354(9):911-923

7 Clifford DB, De Luca A, Simpson DM, Arendt G, Giovannoni G, Nath A. Natalizumab-associated progressive multifocal leukoencephalopathy in patients with multiple sclerosis: lessons from 28 cases. Lancet Neurol 2010;9(4):438-446

8 Kappos L, Radue EW, O'Connor P, et al; FREEDOMS Study Group. A placebo-controlled trial of oral fingolimod in relapsing multiple sclerosis. N Engl J Med 2010;362(5):387-401

9 Cohen JA, Barkhof F, Comi G, et al; TRANSFORMS Study Group. Oral fingolimod or intramuscular interferon for relapsing multiple sclerosis. N Engl J Med 2010;362(5):402-415

10 O'Connor P, Wolinsky JS, Confavreux C, et al; TEMSO Trial Group. Randomized trial of oral teriflunomide for relapsing multiple sclerosis. N Engl J Med 2011;365(14):1293-1303

11 Genzyme Corp. Aubagio (Teriflunomide). Highlights of Prescribing Information. Available at: http://products.sanofi.us/aubagio/aubagio.pdf. Accessed September 14, 2012

12 Schweckendiek W. Heilung von Psoriasis vulgaris. [Treatment of psoriasis vulgaris]. Med Monatsschr 1959;13(2):103-104

13 Schweckendiek W. Behandlung von psoriasis mit lipoidloslichen fumarsaureverbindungen. Medizin Heute 1966;15:219-220

14 Schafer G. Fumarsaure lindert die schuppenflechte. Selecta 1984;15:1260-1261

15 Nugteren-Huying WM, van der Schroeff JG, Hermans J, Suurmond D. Fumaric acid therapy for psoriasis: a randomized, double-blind, placebo-controlled study. J Am Acad Dermatol 1990;22(2 Pt 1): 311-312
16 Altmeyer PJ, Matthes U, Pawlak F, et al. Antipsoriatic effect of fumaric acid derivatives. Results of a multicenter double-blind study in 100 patients. J Am Acad Dermatol 1994;30(6):977-981

17 Hoefnagel JJ, Thio HB, Willemze R, Bouwes Bavinck JN. Long-term safety aspects of systemic therapy with fumaric acid esters in severe psoriasis. Br J Dermatol 2003;149(2):363-369

18 Nieboer C, de Hoop D, Langendijk PN, van Loenen AC, Gubbels J. Fumaric acid therapy in psoriasis: a double-blind comparison between fumaric acid compound therapy and monotherapy with dimethylfumaric acid ester. Dermatologica 1990;181 (1):33-37

19 Werdenberg D, Joshi R, Wolffram S, Merkle HP, Langguth P. Presystemic metabolism and intestinal absorption of antipsoriatic fumaric acid esters. Biopharm Drug Dispos 2003;24(6):259-273

20 Litjens NH, Burggraaf J, van Strijen E, et al. Pharmacokinetics of oral fumarates in healthy subjects. Br J Clin Pharmacol 2004;58 (4):429-432

21 Rostami-Yazdi M, Clement B, Mrowietz U. Pharmacokinetics of anti-psoriatic fumaric acid esters in psoriasis patients. Arch Dermatol Res 2010;302(7):531-538

22 Mrowietz U, Christophers E, Altmeyer P; The German Fumaric Acid Ester Consensus Conference. Treatment of severe psoriasis with fumaric acid esters: scientific background and guidelines for therapeutic use. The German Fumaric Acid Ester Consensus Conference. Br J Dermatol 1999;141(3):424-429

23 Rostami-Yazdi M, Clement B, Schmidt TJ, Schinor D, Mrowietz U. Detection of metabolites of fumaric acid esters in human urine: implications for their mode of action. J Invest Dermatol 2009;129 (1):231-234

24 Schilling S, Goelz S, Linker R, Luehder F, Gold R. Fumaric acid esters are effective in chronic experimental autoimmune encephalomyelitis and suppress macrophage infiltration. Clin Exp Immunol 2006;145(1):101-107

25 Linker RA, Lee DH, Ryan S, et al. Fumaric acid esters exert neuroprotective effects in neuroinflammation via activation of the Nrf2 antioxidant pathway. Brain 2011;134(Pt 3):678-692

26 Duffy S, So A, Murphy TH. Activation of endogenous antioxidant defenses in neuronal cells prevents free radical-mediated damage. J Neurochem 1998;71(1):69-77

27 Scannevin RH, Chollate S, Jung MY, et al. Fumarates promote cytoprotection of central nervous system cells against oxidative stress via the nuclear factor (erythroid-derived 2)-like 2 pathway. J Pharmacol Exp Ther 2012;341(1):274-284

28 Lin SX, Lisi L, Dello Russo C, et al. The anti-inflammatory effects of dimethyl fumarate in astrocytes involve glutathione and haem oxygenase-1. ASN Neuro 2011;3(2):

29 Gilgun-Sherki Y, Melamed E, Offen D. The role of oxidative stress in the pathogenesis of multiple sclerosis: the need for effective antioxidant therapy. J Neurol 2004;251(3):261-268

30 Haider L, Fischer MT, Frischer JM, et al. Oxidative damage in multiple sclerosis lesions. Brain 2011;134(Pt 7):1914-1924

31 van Horssen J, Schreibelt G, Drexhage J, et al. Severe oxidative damage in multiple sclerosis lesions coincides with enhanced antioxidant enzyme expression. Free Radic Biol Med 2008;45 (12):1729-1737

32 Srinivasan R, Ratiney H, Hammond-Rosenbluth KE, Pelletier D, Nelson SJ. MR spectroscopic imaging of glutathione in the white and gray matter at $7 \mathrm{~T}$ with an application to multiple sclerosis. Magn Reson Imaging 2010;28(2):163-170

33 van Horssen J, Drexhage JA, Flor T, Gerritsen W, van der Valk P, de Vries HE. Nrf2 and DJ1 are consistently upregulated in inflammatory multiple sclerosis lesions. Free Radic Biol Med 2010;49 (8):1283-1289

34 Johnson DA, Amirahmadi S, Ward C, Fabry Z, Johnson JA. The absence of the pro-antioxidant transcription factor Nrf2 exacerbates experimental autoimmune encephalomyelitis. Toxicol Sci 2010;114(2):237-246 
35 Moharregh-Khiabani D, Blank A, Skripuletz T, et al. Effects of fumaric acids on cuprizone induced central nervous system deand remyelination in the mouse. PLoS ONE 2010;5(7):e11769

36 Ellrichmann G, Petrasch-Parwez E, Lee DH, et al. Efficacy of fumaric acid esters in the R6/2 and YAC128 models of Huntington's disease. PLoS ONE 2011;6(1):e16172

37 Arnold HM, Huang C, Huang R, et al. Neuroprotective effects of bg12 on malonate-induced striatal lesion volume in Sprague-Dawley rat brain. Neurology 2012;78;01P02121

38 Ghoreschi K, Mrowietz U, Röcken M. A molecule solves psoriasis? Systemic therapies for psoriasis inducing interleukin 4 and Th2 responses. J Mol Med (Berl) 2003;81(8):471-480

39 Frohman EM, Racke MK, Raine CS. Multiple sclerosis-the plaque and its pathogenesis. N Engl J Med 2006;354(9):942-955

40 Thio HB, Zomerdijk TP, Oudshoorn C, et al. Fumaric acid derivatives evoke a transient increase in intracellular free calcium concentration and inhibit the proliferation of human keratinocytes. $\mathrm{Br} \mathrm{J}$ Dermatol 1994;131(6):856-861

41 de Jong R, Bezemer AC, Zomerdijk TP, van de Pouw-Kraan T, Ottenhoff TH, Nibbering PH. Selective stimulation of T helper 2 cytokine responses by the anti-psoriasis agent monomethylfumarate. Eur J Immunol 1996;26(9):2067-2074

42 Asadullah K, Schmid H, Friedrich M, et al. Influence of monomethylfumarate on monocytic cytokine formation-explanation for adverse and therapeutic effects in psoriasis? Arch Dermatol Res 1997;289(11):623-630

43 Zoghi S, Amirghofran Z, Nikseresht A, Ashjazadeh N, KamaliSarvestani E, Rezaei N. Cytokine secretion pattern in treatment of lymphocytes of multiple sclerosis patients with fumaric acid esters. Immunol Invest 2011;40(6):581-596

44 Litjens NH, Rademaker M, Ravensbergen B, Thio HB, van Dissel JT, Nibbering PH. Effects of monomethylfumarate on dendritic cell differentiation. Br J Dermatol 2006;154(2):211-217

45 Vandermeeren M, Janssens S, Borgers M, Geysen J. Dimethylfumarate is an inhibitor of cytokine-induced E-selectin, VCAM-1, and ICAM-1 expression in human endothelial cells. Biochem Biophys Res Commun 1997;234(1):19-23

46 Zhu KJ, Mrowietz U. Inhibition of dendritic cell differentiation by fumaric acid esters. J Invest Dermatol 2001;116(2):203-208

47 Litjens NH, Rademaker M, Ravensbergen B, et al. Monomethylfumarate affects polarization of monocyte-derived dendritic cells resulting in down-regulated Th1 lymphocyte responses. Eur J Immunol 2004;34(2):565-575

48 Peng H, Guerau-de-Arellano M, Mehta VB, et al. Dimethyl fumarate inhibits dendritic cell maturation via nuclear factor $\mathrm{\kappa B}(\mathrm{NF}-\mathrm{KB})$ and extracellular signal-regulated kinase 1 and 2 (ERK1/2) and mitogen stress activated kinase 1 (MSK1) signaling. J Biol Chem 2012;287(33):28017-28026

49 Ghoreschi K, Brück J, Kellerer C, et al. Fumarates improve psoriasis and multiple sclerosis by inducing type II dendritic cells. J Exp Med 2011;208(11):2291-2303

50 Treumer F, Zhu K, Gläser R, Mrowietz U. Dimethylfumarate is a potent inducer of apoptosis in human T cells. J Invest Dermatol 2003;121(6):1383-1388

51 Schimrigk S, Brune N, Hellwig K, et al. Oral fumaric acid esters for the treatment of active multiple sclerosis: an open-label, baseline-controlled pilot study. Eur J Neurol 2006;13(6): 604-610

52 Fox RJ, Miller DH, Phillips JT, et al; CONFIRM Study Investigators. Placebo-controlled phase 3 study of oral BG-12 or glatiramer in multiple sclerosis. N Engl J Med 2012;367(12):1087-1097

53 Gold R, Kappos L, Arnold DL, et al; DEFINE Study Investigators. Placebo-controlled phase 3 study of oral BG-12 for relapsing multiple sclerosis. N Engl J Med 2012;367(12):1098-1107

54 Talalay P, De Long MJ, Prochaska HJ. Identification of a common chemical signal regulating the induction of enzymes that protect against chemical carcinogenesis. Proc Natl Acad Sci U S A 1988;85 (21):8261-8265
55 Friling RS, Bensimon A, Tichauer Y, Daniel V. Xenobiotic-inducible expression of murine glutathione S-transferase Ya subunit gene is controlled by an electrophile-responsive element. Proc Natl Acad Sci U S A 1990;87(16):6258-6262

56 Rushmore TH, Pickett CB. Transcriptional regulation of the rat glutathione S-transferase Ya subunit gene. Characterization of a xenobiotic-responsive element controlling inducible expression by phenolic antioxidants. J Biol Chem 1990;265(24):14648-14653

57 Itoh K, Mimura J, Yamamoto M. Discovery of the negative regulator of Nrf2, Keap1: a historical overview. Antioxid Redox Signal 2010;13(11):1665-1678

58 Li W, Kong AN. Molecular mechanisms of Nrf2-mediated antioxidant response. Mol Carcinog 2009;48(2):91-104

59 Takaya K, Suzuki T, Motohashi H, et al. Validation of the multiple sensor mechanism of the Keap1-Nrf2 system. Free Radic Biol Med 2012;53(4):817-827

60 Brigelius-Flohé R, Flohé L. Basic principles and emerging concepts in the redox control of transcription factors. Antioxid Redox Signal 2011;15(8):2335-2381

61 Schmidt TJ, Ak M, Mrowietz U. Reactivity of dimethyl fumarate and methylhydrogen fumarate towards glutathione and N-acetyl-Lcysteine-preparation of S-substituted thiosuccinic acid esters. Bioorg Med Chem 2007;15(1):333-342

62 Lehmann JCU, Listopad JJ, Rentzsch CU, et al. Dimethylfumarate induces immunosuppression via glutathione depletion and subsequent induction of heme oxygenase 1 . J Invest Dermatol 2007;127(4):835-845

63 Schmidt MM, Dringen R. Fumaric acid diesters deprive cultured primary astrocytes rapidly of glutathione. Neurochem Int 2010;57 (4):460-467

64 Thiessen A, Schmidt MM, Dringen R. Fumaric acid dialkyl esters deprive cultured rat oligodendroglial cells of glutathione and upregulate the expression of heme oxygenase 1 . Neurosci Lett 2010;475(1):56-60

65 Gerdes S, Shakery K, Mrowietz U. Dimethylfumarate inhibits nuclear binding of nuclear factor kappaB but not of nuclear factor of activated $\mathrm{T}$ cells and CCAAT/enhancer binding protein beta in activated human T cells. Br J Dermatol 2007;156(5):838-842

66 Gesser B, Johansen C, Rasmussen MK, et al. Dimethylfumarate specifically inhibits the mitogen and stress-activated kinases 1 and 2 (MSK1/2): possible role for its anti-psoriatic effect. J Invest Dermatol 2007;127(9):2129-2137

67 Bista P, Zeng WK, Ryan S, Lukashev M, Yamamoto M. Dimethyl fumarate suppresses inflammation in vitro via both nrf2-dependent and nrf2-independent pathways. Neurology 2012;78(Meeting Abstracts 1):P02.108

68 Hanson J, Gille A, Zwykiel S, et al. Nicotinic acid- and monomethyl fumarate-induced flushing involves GPR109A expressed by keratinocytes and COX-2-dependent prostanoid formation in mice. J Clin Invest 2010;120(8):2910-2919

69 Sheikh SI, Nestorov I, Russell H, et al. Safety, tolerability, and pharmacokinetics of bg-12 administered with and without aspirin: key findings from a randomized, double-blind, placebo-controlled trial in healthy volunteers [abstract]. Neurology 2012;78 (Meeting Abstracts 1): P04.136

70 Kappos L, Gold R, Miller DH, et al; BG-12 Phase IIb Study Investigators. Efficacy and safety of oral fumarate in patients with relapsing-remitting multiple sclerosis: a multicentre, randomised, double-blind, placebo-controlled phase IIb study. Lancet 2008;372 (9648):1463-1472

71 Comi G, Filippi M, Wolinsky JS; European/Canadian Glatiramer Acetate Study Group. European/Canadian multicenter, doubleblind, randomized, placebo-controlled study of the effects of glatiramer acetate on magnetic resonance imaging-measured disease activity and burden in patients with relapsing multiple sclerosis. Ann Neurol 2001;49(3):290-297

72 Gold R, Miller DH, Phillips JT, et al. Clinical efficacy of BG-12 in relapsing-remitting multiple sclerosis: an integrated analysis of 
the phase 3 DEFINE and CONFIRM studies. Paper presented at: 28th Congress of the European Committee for Treatment and Research in Multiple Sclerosis; October 10-13, 2012; Lyon, France 73 Miller D, Gold R, Fox RJ, et al. Effects of BG-12 on magnetic resonance imaging outcomes in relapsing-remitting multiple sclerosis: an integrated analysis of the phase 3 define and confirm studies. Paper presented at: 28th Congress of the European Committee for Treatment and Research in Multiple Sclerosis; October 10-13, 2012; Lyon, France

74 Sormani MP, Bonzano L, Roccatagliata L, Cutter GR, Mancardi GL, Bruzzi P. Magnetic resonance imaging as a potential surrogate for relapses in multiple sclerosis: a meta-analytic approach. Ann Neurol 2009;65(3):268-275

75 Mikol DD, Barkhof F, Chang P, et al; REGARD study group. Comparison of subcutaneous interferon beta-1a with glatiramer acetate in patients with relapsing multiple sclerosis (the REbif vs Glatiramer Acetate in Relapsing MS Disease [REGARD] study): a multicentre, randomised, parallel, open-label trial. Lancet Neurol 2008;7(10):903-914
76 Cadavid D, Wolansky LJ, Skurnick J, et al. Efficacy of treatment of MS with IFNbeta-1b or glatiramer acetate by monthly brain MRI in the BECOME study. Neurology 2009;72(23): 1976-1983

77 O'Connor P, Arnason B, Comi G, et al. Interferon beta-1b 500 mcg, interferon beta-1b $250 \mathrm{mcg}$ and glatiramer acetate: primary outcomes of the Betaferon ${ }^{\circledR} /$ Betaseron ${ }^{\circledR}$ efficacy yielding outcomes of a new dose study. Neurology 2008;70(11):LBS.004

78 Calabresi P, Radue EW, Goodin D, et al. Efficacy and safety of fingolimod in patients with relapsing-remitting multiple sclerosis (RRMS): Results from an additional 24-month double-blind, placebo-controlled study (FREEDOMS II study). Neurology 2012;79: e90-e91

79 Kappos L, Comi G, Confavreux C, et al. The efficacy and safety of teriflunomide in patients with relapsing MS: Results from TOWER, a phase III, placebo-controlled study (abstract). Abstract presented at: 28th Congress of the European Committee for Treatment and Research in Multiple Sclerosis; October 9-13, 2012; Lyon, France 